\title{
Médiévales
}

Langues, Textes, Histoire

59 | automne 2010

Théâtres du Moyen Âge

\section{Les origines de la légende arthurienne : six théories}

The origins of the Arthurian legend: six theories

\section{Mark Adderley et Alban Gautier}

\section{(2) OpenEdition}

Journals

Édition électronique

URL : https://journals.openedition.org/medievales/6173

DOI : 10.4000/medievales.6173

ISSN : $1777-5892$

Éditeur

Presses universitaires de Vincennes

Édition imprimée

Date de publication : 31 décembre 2010

Pagination : 183-193

ISBN : 978-2-84292-267-2

ISSN : 0751-2708

Référence électronique

Mark Adderley et Alban Gautier, «Les origines de la légende arthurienne : six théories », Médiévales [En ligne], 59 | automne 2010, mis en ligne le 20 mars 2013, consulté le 22 avril 2022. URL : http:// journals.openedition.org/medievales/6173; DOI : https://doi.org/10.4000/medievales.6173 
Médiévales 59, automne 2010, p. 183-193

Mark ADDERLEY

Alban GAUTIER

\section{LES ORIGINES DE LA LÉGENDE ARTHURIENNE : SIX THÉORIES}

Tous les spécialistes qui se sont intéressés de manière honnête et sérieuse aux origines de la légende arthurienne se plaisent à rappeler que les grands textes qui font la matière de Bretagne, ceux qui nous racontent le roi Arthur, sont tardifs: les plus anciens datent du début du XII ${ }^{\mathrm{e}}$ siècle, et presque tous s'inspirent du récit pseudo-historique proposé au milieu des années 1130 par Geoffroy de Monmouth à travers son Histoire des rois de Bretagne ${ }^{1}$. Le plus ancien texte «historique» qui mentionne le personnage, et encore de manière relativement sommaire, est l'Historia Brittonum attribuée (à tort) au moine Nennius, qui remonterait à la première moitié $\mathrm{du} \mathrm{IX}^{\mathrm{e}}$ siècle, soit environ trois siècles après les faits qu'il prétend relater; aucune source contemporaine des événements, c'est-à-dire produite entre 400 et 600 , ne fait mention d'un Arthur; et les autres sources antérieures à Geoffroy, composées entre le $\mathrm{IX}^{\mathrm{e}}$ et le début du XII ${ }^{\mathrm{e}}$ siècle, restent pour la plupart très laconiques.

À ceux qui se penchent sur la question, Arthur semble introuvable. Histoire ou légende? La question semble sans issue. Faut-il alors abandonner toute velléité de trouver Arthur? Pourquoi s'intéresser plus longtemps à une question cent fois rebattue, qui pourrait de fait sembler sans importance? La plupart des historiens écrivant aujourd'hui sur la période évitent soigneusement de le mentionner, ou le rejettent en une ou deux phrases dans les marges de l'histoire «scientifique». C'est le cas de la plupart des grandes ou petites synthèses parues depuis une trentaine d'années: on peut mentionner, parmi une production très abondante, les ouvrages de Barbara Yorke, de Ken Dark, de

1. N. Wright éd., The Historia Regum Britannie of Geoffrey of Monmouth, vol. 1 : Text, Cambridge, 1985 ; L. Mathey-Maille éd., Geoffroy de Monmouth: Histoire des rois de Bretagne, Paris, 1993. 
Thomas Charles-Edwards ou de Stéphane Lebecq ${ }^{2}$. Les deux plus importantes de ces synthèses, les plus influentes, qui restent aujourd'hui les manuels de base pour la connaissance de la période, sont sans nul doute le livre de Peter Salway sur la Bretagne romaine (paru en 1981) et le volume sur les Anglo-Saxons dirigé par James Campbell (paru l'année suivante): toutes deux ont été rééditées de nombreuses fois et, sans toutefois se pencher de manière exhaustive sur les $\mathrm{V}^{\mathrm{e}}-\mathrm{VI}^{\mathrm{e}}$ siècles, présentent une image complète et documentée des deux périodes qui l'encadrent. Or, Salway comme Campbell rejettent également Arthur hors de l'écriture historique sérieuse. Le premier suspend son jugement: «son existence même est une question que les historiens doivent laisser de côté, en attendant que la valeur des textes ait été réexaminée par les spécialistes habilités à le faire ${ }^{3}$ ». Le second va quant à lui jusqu'à condamner la question comme sans pertinence et parle de «l'intérêt inépuisable, mais plutôt ridicule, à chercher qui était le "vrai" Arthur», ajoutant cependant que cela «force à prendre conscience du nombre de grands hommes et de grands événements des $v^{\mathrm{e}}$ et $\mathrm{VI}^{\mathrm{e}}$ siècles, dont nous ne savons absolument rien ${ }^{4} »$.

Or, depuis les années 1950, de nombreux médiévistes, professionnels et amateurs, historiens, archéologues ou littéraires, ont tenté de rendre compte des origines de la légende arthurienne, sans ridicule ni malhonnêteté 5 . Les approches ont été diverses, et les conclusions encore plus. Nous tenterons ici, pour les résumer, de les répartir en six théories ou groupes de théories ${ }^{6}$, couvrant tout le spectre des publications scientifiques sérieuses. Ont été exclues les théories trop fantaisistes, voire fantastiques, qui font florès en la matière; on remarquera par

2. B. Yorke, Kings and Kingdoms of Early Anglo-Saxon England, Londres et New York, 1990, p. 2; K.R. DARK, Civitas to Kingdom : British Political Continuity, Londres, 1999; T. CharLesEdwards éd., After Rome, Oxford, 2003 (Short Oxford History of the British Isles, 2), p. 29; S. LeBECQ éd., Histoire des îles Britanniques, PUF, 2007, p. 56.

3. P. Salway, A History of Roman Britain, Oxford, 1997, p. 485.

4. J. CampBell éd., The Anglo-Saxons, Londres, 1982, p. 27.

5. Un grand nombre de sources sont par ailleurs disponibles, et la plupart du temps discutées, dans divers anthologies et ouvrages critiques. E. K. CHAMBERs, Arthur of Britain, Londres, 1927, présente les principaux textes; E. FARAL, La Légende arthurienne. Études et documents. Les plus anciens textes, Paris, 1929, contient le texte de l'ensemble des sources latines anciennes, y compris les textes de Gildas et de Geoffroy de Monmouth; L. Fleuriot, J.-C. Lozac'hmeur et L. Prat, Récits et poèmes celtiques. Domaine brittonique, $v t^{e}-X V^{e}$ siècles, Paris, 1981, est une anthologie assez variée de la littérature galloise et bretonne d'Armorique, pas exclusivement arthurienne; J. B. CoE et S. Young, The Celtic Sources for the Arthurian Legend, Llanerch, 1995, est une anthologie plus ou moins exhaustive de la matière brittonique, en traduction anglaise.; C. SNYDER, An Age of Tyrants: Britain and the Britons, A.D. 400-600, University Park (PA), 1998, est un catalogue commenté des sources concernant le $\mathrm{V}^{\mathrm{e}}$ - $\mathrm{vl}^{\mathrm{e}}$ siècle en Grande-Bretagne.

6. Une première version de cet article, distinguant cinq théories, a été mise en ligne par Mark Adderley sur son site personnel:

http://www.markadderley.net/arthur/historical-arthur/historical-arthur.html. 
ailleurs que les théories $n^{\circ} 3$ et $n^{\circ} 4$ ont été fortement critiquées et n'ont généralement pas été retenues par les spécialistes de la période. Pour chaque théorie, nous proposons un résumé de la théorie, nous rappelons les principaux arguments qui la sous-tendent et nous indiquons les principaux ouvrages et articles qui l'ont défendue; enfin, nous donnons un aperçu de la réception de cette théorie.

\section{Théorie $\mathrm{n}^{\circ} 1$ : Arthur, chef de guerre du v $\mathrm{v}^{\mathrm{e}}-\mathrm{vI}^{\mathrm{e}}$ siècle}

\section{Résumé de la théorie}

Arthur était une figure militaire majeure du $\mathrm{v}^{\mathrm{e}}$ siècle finissant, luttant contre les Saxons, et peut-être aussi contre les Pictes, dans les années 490-500. Il remporta sa plus grande victoire sur les Saxons au mont de Badon, dans le Sud-Ouest de la (Grande-) Bretagne. Il mourut peut-être une vingtaine d'années plus tard, après une période de paix relative, dans une guerre civile. À la fin du $\mathrm{VI}^{\mathrm{e}}$ siècle, son nom était toujours dans les esprits, et plusieurs enfants le reçurent; il devint peu à peu une figure importante de la littérature galloise.

\section{Arguments}

1. Dans son De Excidio et conquestu Britanniae 7 (De la chute et de la conquête de la Bretagne), le moine Gildas rappelle les étapes du conflit entre les Saxons et les Bretons, menés par un certain Ambrosius Aurelianus : ce conflit aurait culminé dans la bataille du mons Badonicus, peut-être autour de 500. Mais Gildas ne donne pas le nom du chef des Bretons lors de cette bataille.

2. Un poème héroïque et élégiaque, $Y$ Gododdin (aussi connu sous le titre de Canu Aneirin ${ }^{8}$ ), composé par le barde gallois Aneirin vers 600, compare un guerrier nommé Guaurthur à Arthur; vers 600, le nom d'Arthur est présent dans quatre familles royales de l'Ouest de l'île. Il est donc probable que vers 600, Arthur ait déjà été célèbre.

3. L'Historia Brittonum (Histoire des Bretons) du pseudo-Nennius ${ }^{9}$, écrite vers 830 , est une collection de documents se rapportant au folklore et à l'histoire de la Bretagne. L'auteur dresse une liste de douze batailles attribuées au dux bellorum (chef des batailles) Arthur, culminant avec celle du mont de Badon.

7. M. Winterbottom éd., Gildas: The Ruin of Britain, Londres et Chichester, 1978 (Arthurian Period Sources, 7).

8. K. H. Jackson éd., The Gododdin, The Oldest Scottish Poem, Édimbourg, 1969.

9. J. Morris éd., Nennius : British History and the Welsh Annals, Londres et Chichester, 1980 (Arthurian Period Sources, 8). 
4. Les Annales Cambriae ${ }^{10}$ (Annales de Galles), compilation anonyme du $\mathrm{x}^{\mathrm{e}}$ siècle, mentionnent pour l'année 518 une bataille de Badon, dans laquelle Arthur aurait porté la croix de Jésus-Christ pendant trois jours et trois nuits, et où les Bretons furent vainqueurs; elles rapportent aussi une bataille de Camlann, dans laquelle Arthur et Medraut (le futur Modred de la légende) tombèrent. Ces annales ont pu être copiées tardivement à partir de tables pascales plus anciennes.

5. Des fouilles archéologiques entreprises à South Cadbury dans le Somerset au milieu des années $1960^{11}$ ont pu montrer que ce site de hauteur, associé à la légendaire Camelot depuis le XVI siècle au moins, avait fait l'objet d'importants travaux de fortification à la fin du $v^{\mathrm{e}}$ siècle.

\section{Bibliographie}

K. H. Jackson, «The Arthur of History», dans R. S. Loomis éd., Arthurian Literature in the Middle Ages: A Collaborative History, Oxford, 1959, p. 1-11.

G. Ashe, «The Arthurian Fact», dans ID. éd., The Quest for Arthur's Britain, Londres, 1968, p. 27-57.

L. Alcock, Arthur's Britain: History and Archaeology, AD 367-634, Londres, 1971.

J. Morris, The Age of Arthur: A History of the British Isles from 350 to 650 , Londres, 1973.

C. GidLow, The Reign of Arthur, Sutton, 2004.

\section{Réception}

Cette théorie, en particulier dans la version raisonnable et intelligente proposée par Leslie Alcock, est devenue la forme «classique» de l'explication historicisante de la légende arthurienne. En revanche, la grandiose construction de John Morris, qui faisait d'Arthur un empereur de Bretagne à la manière romaine, a très vite été dénoncée comme une vaste affabulation. Si la théorie d'Alcock a été critiquée, l'idée d'une «paix arthurienne» au début du $\mathrm{VI}^{\mathrm{e}}$ siècle reste en revanche défendue par de nombreux auteurs.

10. Ibid.

11. L. Alcock, S. J. Stevenson et C. R. Musson, Cadbury Castle, Somerset: The Early Medieval Archaeology, Cardiff, 1995. 


\section{Théorie $n^{\circ} 2$ : Arthur insaisissable}

\section{Résumé de la théorie}

Peut-être y a-t-il eu un Arthur historique, mais nous n'avons aucun moyen de rien connaître à son sujet. Les sources concernant la possible période de son activité, à commencer par le traité de Gildas, sont rares et peu fiables: au mieux, elles peuvent être utilisées pour compléter une trame historique principalement construite à partir de l'archéologie.

\section{Arguments}

1. Gildas ne mentionne pas Arthur; la victoire bretonne de Badon pourrait tout aussi bien être attribuée à Ambrosius Aurelianus que Gildas mentionne à la phrase précédente. Par ailleurs, Gildas n'écrit pas un ouvrage d'histoire mais une admonitio à ses contemporains du milieu du $\mathrm{VI}^{\mathrm{e}}$ siècle: il ne saurait représenter un témoignage fiable sur les événements du siècle précédent.

2. Le Canu Aneirin est trop difficile à dater pour être utile à l'historien: même si le poème a pu être composé pour la première fois à la fin du $\mathrm{vI}^{\mathrm{e}}$ siècle, il n'a été copié que plusieurs siècles après et a pu connaître des transformations profondes et des interpolations, y compris l'ajout du nom d'Arthur.

3. L'auteur de l'Historia Brittonum, quel qu'il soit, ne s'est pas contenté de recopier des textes plus anciens, mais il a prétendu faire œuvre d'historien en faisant concorder des documents contradictoires, produisant ainsi des erreurs; il a aussi fait usage de sources légendaires.

4. Les Annales Cambriae ont été compilées pour la première fois au $\mathrm{VIII}^{\mathrm{e}}$ siècle, et toutes les dates antérieures à 770 ont été complétées a posteriori, en particulier à l'aide de l'Historia Brittonum. Puisqu' Arthur était déjà une figure légendaire à cette époque, nous ne pouvons pas nous appuyer sur ce texte pour confirmer son existence.

\section{Bibliographie}

D. N. Dumville, «Sub-Roman Britain: History and Legend», History, 62, 1977, p. 173-192.

D. N. Dumville, «The Historical Value of the Historia Brittonum», Arthurian Literature, 6, 1986, p. 1-26.

D. N. Dumville, Histories and Pseudo-histories of the Insular Middle Ages, Aldershot, 1990.

T. Charles-Edwards, «The Arthur of History», dans R. Bromwich, A. O. H. Jarman et B. F. Roberts éd., The Arthur of the Welsh: The Arthurian Legend in Medieval Welsh Literature, Cardiff, 1991, p. 15-32. 
A. Gautier, Arthur, Paris, 2007.

A. Chaudu, Le Roi Arthur, Paris, 2009.

\section{Réception}

Cette attitude sceptique s'oppose principalement à ce que David N. Dumville a appelé l'école du «no smoke without fire», c'est-à-dire à ceux qui considèrent que l'existence d'une légende arthurienne signifie nécessairement que celle-ci s'appuie sur un fond de vérité. Dumville et d'autres défendent au contraire l'idée selon laquelle l'historien ne peut parvenir à des conclusions solides sur une période donnée qu'en utilisant des témoignages écrits émanant des contemporains, et que les sources plus tardives ne lui sont d'aucune utilité. La réception de cette critique vigoureuse a été excellente dans les milieux académiques, plus difficile à faire passer auprès du grand public.

\section{Théorie $n^{\circ} 3$ : Arthur, avatar de Riothamus}

\section{Résumé de la théorie}

Arthur était un roi breton, dont l'histoire n'a pas conservé le souvenir par son nom, mais par son titre, Riothamus, en langue brittonique *rigo-tamos, c'est-à-dire « roi suprême ». Appelé en Gaule par l'empereur Anthemius en 468, il combattit les Wisigoths, fut trahi par le préfet Arvandus et connut une ultime défaite en Bourgogne vers 470.

\section{Arguments}

1. La Vie de Goueznou ${ }^{12}$, écrite vers 1019 (c'est-à-dire bien avant l'Histoire des rois de Bretagne de Geoffroy de Monmouth), désigne Arthur comme «roi des Bretons», combattant les Saxons et remportant plusieurs victoires en Bretagne comme en Gaule.

2. Sidoine Apollinaire évoque au milieu du v ve siècle dans une de ses lettres la traîtrise du préfet des Gaules Arvandus, qui aurait dévoilé au roi wisigoth Euric le moyen d'attaquer et de défaire l'armée du roi des Bretons Riothamus.

3. Dans son Histoire des Goths de 551, Jordanès expose comment l'empereur Anthemius appela à son secours le général Riotimos, roi des Bretons. Celui-ci est arrivé depuis l'autre côté de la mer, ce qui signifie qu'il ne venait pas de Bretagne armoricaine.

12. Vita sancti Wohedouii (dans J. CoE et S. Young, The Celtic Sources, op. cit., p. 36). 
4. Grégoire de Tours ${ }^{13}$ désigne la ville de Déols, près de Châteauroux, comme le lieu où furent défaits les Bretons; depuis Déols, Riothamus aurait fui en direction de l'actuelle Bourgogne, allant mourir près d'Avallon, un nom chargé d'évocations arthuriennes.

5. Geoffroy de Monmouth mentionne certains de ces évènements dans son Histoire des rois de Bretagne, mais en les rattachant à Arthur et non à Riothamus. Son récit mentionne un procurateur romain (Lucius), un empereur (Léon), et un pape (Sulpicius) : derrière ceux-ci, Ashe prétend voir l'empereur d'Occident Anthemius (467-472), l'empereur d'Orient Léon I ${ }^{\text {er }}$ (457-474) et le pape Simplicius (468-483).

6. La réoccupation de South Cadbury est contemporaine des ces événements.

\section{Bibliographie}

L. Fleuriot, Les Origines de la Bretagne, Paris, 1980.

G. Ashe, " "A Certain Very Ancient Book": Traces of an Arthurian Source in

Geoffrey of Monmouth's Historia», Speculum, 56, 1981, p. 301-323.

G. Ashe, The Discovery of King Arthur, New York, 1985.

G. AsHe, «The Origins of the Arthurian Legend», Arthuriana, 5, 1995, p. 1-23.

\section{Réception}

Cette théorie a été principalement défendue par Geoffrey Ashe à partir de pistes lancées par Léon Fleuriot, mais elle a été efficacement réfutée par Kenneth Jackson, Ian Wood et d'autres ${ }^{14}$.

\section{Théorie $n^{\circ} 4$ : La piste sarmate}

\section{Résumé de la théorie}

Le personnage qui a inspiré la légende arthurienne est un certain Lucius Artorius Castus, citoyen romain d'origine dalmate, préfet au II ${ }^{\mathrm{e}}$ siècle de notre ère de la $\mathrm{VI}^{\mathrm{e}}$ légion Victrix cantonnée à York. Or, au $\mathrm{III}^{\mathrm{e}}$ siècle, certains auxiliaires de la cette même légion étaient d'origine sarmate, ce qui expliquerait les ressemblan-

13. Grégoire de Tours, Decem Libri Historiarum, I, 18 (trad. R. LATOuche, Histoire des Francs, Paris, rééd. 1999, p.106-107).

14. I. N. Wood, «The Fall of the Western Empire and the End of Roman Britain», Britannia, 18 , 1987, p. 251-262, sp. p. 261-262; O. J. PADEL, «Recent Work on the Origins of the Arthurian Legend: A Comment», Arthuriana, 5, 1995, p. 103-114. 
ces étonnantes entre la légende arthurienne et les croyances et mythes des descendants actuels des Sarmates, à savoir les Ossètes étudiés entre autres par Georges Dumézil ${ }^{15}$.

\section{Arguments}

1. À la différence des autres soldats romains, les Sarmates étaient des cavaliers protégés par une armure, tout comme les chevaliers d'Arthur.

2. Ils utilisaient un étendard en forme de dragon, tout comme Arthur, fils d'Uther Pendragon, sur certaines illustrations médiévales.

3. Ils vénéraient une épée nue fichée en terre, qui rappelle l'histoire de l'épée dans le perron.

4. Ils inhalaient du chanvre bouillonnant dans un chaudron, qui pourrait correspondre au Graal.

5. Le récit des derniers instants d'Arthur dans la Mort le roi Artu (début du XIII ${ }^{\mathrm{e}}$ siècle) suit de manière saisissante la trame narrative de la mort du héros ossète Batradz. Dans les deux cas, le héros mourant demande que son épée soit jetée dans un lac: ses compagnons tentent d'abord de garder l'épée, mais devant l'insistance du héros, ils doivent se résoudre à s'en défaire, provoquant un phénomène extraordinaire qui trouble la surface de l'eau (surgissement d'une main dans le cas d'Arthur, tourbillon et tempête dans le cas de Batradz).

\section{Bibliographie}

K. Malone, «Artorius», Modern Philology, 22, 1925, p. 367-374.

J. GRISWARD, «Le motif de l'épée jetée au lac: la mort d'Arthur et la mort de Batradz», Romania, 90, 1969, p. 289-340 et p. 473-514.

H. NicKEL, «The Dawn of Chivalry», dans From the Lands of the Scythians: Ancient Treasures from the Museums of the USSR, 3000 BC-100 BC, New York, 1975.

C. S. Littleton et A. C. Thomas, «The Sarmatian Connection: New Light on the Origin of the Arthurian and Holy Grail Legends », Journal of American Folklore, 91, 1978, p. 513-527.

C. S. Littletonet L.A. Malcor, From Scythia to Camelot: ARadical Reassessment of the Legends of King Arthur, the Knights of the Round Table, and the Holy Grail, New York, 1994.

15. G. DuméziL, Le Livre des héros. Légendes sur les Nartes, Paris, 1965. 


\section{Réception}

Kemp Malone a exhumé dès les années 1920 le personnage d'Artorius, Joël Grisward a mis en lumière les ressemblances entre légendes ossètes et matière de Bretagne, et Scott Littleton a relié les deux bouts de la chaîne. Si elle a eu ses défenseurs, cette théorie est aujourd'hui abandonnée: nul besoin d'aller chercher Arthur chez les auxiliaires sarmates du III siècle, ni de l'historiciser à travers la figure d'un officier dalmate du $\mathrm{II}^{\mathrm{e}}$ siècle - et encore moins les deux à la fois.

\section{Théorie $n^{\circ} 5$ : Arthur du Nord}

\section{Résumé de la théorie}

Qu'il soit ou non un personnage historique, Arthur est essentiellement une figure du Nord de la Grande-Bretagne, voire une figure écossaise, adoptée tardivement dans le monde gallois ou armoricain. S'il faut le chercher quelque part, c'est plutôt dans l'ancienne Calédonie, ou aux alentours du mur d'Hadrien.

\section{Arguments}

1. La plupart des batailles mentionnées par le pseudo-Nennius pourraient être localisées dans le Nord: c'est le cas de la bataille de la «forêt calédonienne», au nom éloquent.

2. Le Canu Aneirin, qui peut être considéré comme le plus ancien texte mentionnant Arthur, commémore précisément une bataille ayant impliqué l'un de ces peuples bretons du Nord, les Gododdin de la région d'Édimbourg. La langue du poème est certes galloise, mais la matière en est nordique.

3. Le $\mathrm{IX}^{\mathrm{e}}$ siècle, moment où fut rédigée l'Historia Britonnum, correspond à une période de grande popularité des héros nordiques comme Merlin ou comme le guerrier Cunedda, à qui les souverains gallois de Gwynedd ont cherché à rattacher leur généalogie.

\section{Bibliographie}

T. Jones, «The Early Evolution of the Legend of Arthur», Nottingham Medieval Studies, 8, 1964, p. 3-21.

R. Bromwich, «Concepts of Arthur», Studia Celtica, 10-11, 1975-1976, p. 163181.

A. O. H. Jarman, «The Arthurian Allusions in the Book of Aneirin», Studia Celtica, 24-25, 1989-1990, p. 15-25. 


\section{Réception}

D'abord plutôt bien reçue, cette théorie est aujourd'hui assez peu relayée. Elle se heurte en effet à un grand nombre d'obstacles, dont le moindre n'est pas la localisation de la bataille du mont de Badon, généralement située dans le SudOuest de l'île. Par ailleurs, les lieux que l'Historia Brittonum relie à Arthur se trouvent plutôt dans le Sud-Est du pays de Galles ou dans les marches anglo-galloises, des régions distinctes du Gwynedd où cette matière nordique a été incorporée à la tradition littéraire galloise.

\section{Théorie $n^{\circ} 6$ : Arthur, héros mythologique et figure folklorique}

\section{Résumé de la théorie}

Arthur n'est en aucun cas un personnage historique. C'est une figure folklorique, dérivée de la mythologie celtique, historicisée par des auteurs comme le pseudo-Nennius puis Geoffroy de Monmouth. Plus précisément, il pourrait s'agir d'un héros ours, dernier avatar d'une divinité celtique ayant fourni matière à légende dans de nombreuses régions du monde britonnique, au pays de Galles comme en Bretagne armoricaine ou sur les marches angloécossaises.

\section{Arguments}

1. De nombreux auteurs médiévaux historicisent des héros folkloriques: ce n'est pas parce que le pseudo-Nennius fait d'Arthur un personnage historique qu'il en était effectivement un.

2. Les sources les plus explicitement «historiques» l'évoquent elles aussi dans un contexte folklorique et merveilleux. Le pseudo-Nennius mentionne ainsi le chien et le fils du miles Arthur et les relie à des phénomènes naturels inexpliqués. L'Historia Brittonum et les Annales Cambriae lui attribuent des exploits proprement extravagants, comme le massacre de centaines d'ennemis.

3. L'immense majorité des textes produits avant le XII siècle, et encore une grande partie de ceux qui ont succédé à Geoffroy de Monmouth, placent Arthur dans des situations entièrement légendaires, mythiques ou folkloriques. Les plus anciens textes gallois, qu'ils soient en latin comme les Vies de saint Cadoc et de saint Padarn, ou en gallois comme les Dépouilles d'Annwfyn, Pa gur, Culhwch et Olwen et les Triades de l'île de Bretagne, font en effet d'Arthur un héros tueur de monstres, combattant contre des loups-garous, des chats monstrueux ou des sorcières bien plus que contre des Saxons historiques. 
4. La racine celtique art- signifie «ours». Le nom brittonique (non attesté) *arto-wiros, signifiant littéralement «ours-homme», pourrait être l'origine du nom d'Arthur. Or, de nombreux éléments de la légende arthurienne rappellent cette identité ursine du roi : son dernier sommeil sous la terre semblable à l'hibernation de l'ours, la manière dont il tue ses ennemis par étouffement (chez Geoffroy de Monmouth).

\section{Bibliographie}

O. J. PADEL, «The Nature of Arthur», Cambrian Medieval Celtic Studies, 27, 1994, p. 1-31.

O. J. PADEL, Arthur in Medieval Welsh Literature, Cardiff, 2000.

T. GREEN, «The Historicity and Historicization of Arthur», Arthurian Resources, 2001. Publication électronique:

$<$ http://www.arthuriana.co.uk/historicity/arthur.htm >

P. WALTer, Arthur. L'ours et le roi, Paris, 2002.

N. J. Higham, King Arthur. Myth-Making and History, Londres et New York, 2002.

M. Aurell, La Légende du roi Arthur, Paris, 2007.

T. Green, Concepts of Arthur : Early Arthurian Tradition and the Origins of the Legend, Stroud, 2007.

\section{Réception}

Comme le montrent les dates de parution de la plupart des ouvrages, cette dernière théorie est l'une des plus en vogue à ce jour, et l'une des plus solidement étayées. N. J. Higham a critiqué certains aspects de l'identification d'Arthur à une divinité ursine celtique, tout en conservant l'essentiel de l'argumentaire qui voit en lui une figure essentiellement mythologique tardivement historicisée.

Mark Adderley - Wyoming Catholic College, P.O. Box 750, Lander, WY 82520, États-Unis

Alban Gautier - Université du Littoral Côte d'Opale, 34 Grande Rue, BP 751, 62321 Boulogne-sur-Mer Cedex 